Artigo original reportando investigação clínica ou básica

\title{
A PRESENÇA DA FAMÍLIA NOS HOSPITAIS EM TEMPOS DE PANDEMIA E O PARADOXO DA SEGURANÇA DO DOENTE
}

THE FAMILY'S PRESENCE IN HOSPITALS IN PANDEMIC TIME AND THE PATIENT SAFETY PARADOX

LA PRESENCIA FAMILIAR EN LOS HOSPITALES EN TIEMPO DE PANDEMIA Y LA PARADOJA DE LA SEGURIDAD DEL PACIENTE

DOI 10.33194/rper.2021.176; Data de Receção: 2021-06-01; Data de Aceitação: 2021-10-18

\author{
Tânia Correia ${ }^{1}$ (D); Maria Manuela Martins ${ }^{1,2}$ (D); Fernando Barroso ${ }^{3} \mathbb{D}$ \\ ${ }^{1}$ ICBAS e NursID - CINTESIS, Porto, Portugal; ${ }^{2}$ Escola Superior de Enfermagem do Porto, Porto, Portugal; \\ ${ }^{3}$ Centro Hospitalar de Setúbal (HS), DGS, Setúbal, Portugal
}

\author{
Autor correspondente: Tânia Correia, tsp.correia@gmail.com
}

\section{RESUMO}

Introdução: Devido à pandemia por COVID-19, para garantir a segurança dos cuidados de saúde e conter a sua transmissão, foram adotadas medidas restritivas que afastaram as famílias dos doentes hospitalizados. Tais medidas trouxeram implicações.

Objetivo: analisar, a partir dos discursos dos enfermeiros especialistas em enfermagem de reabilitação (EEER), as vantagens, desvantagens e contextos da presença da família nos hospitais, à luz da segurança do doente, em contexto pandémico.

Metodologia: Estudo qualitativo interpretativo fundamentado no modelo de risco de James Reason, realizado através de entrevista semiestruturada a 6 EEER escolhidos por conveniência. Realizada análise de conteúdo com recurso ao software Atlas.ti. e à metodologia de Bardin.

Resultados: Foram identificadas 17 categorias agrupadas de acordo com a representação da família na segurança do doente: 10 categorias na visão da família potencializadora de falhas de segurança e 7 no âmbito da família como barreira de segurança.

Discussão: Os EEER consideram a presença da família como muito importante para doentes hospitalizados e para a segurança dos cuidados., No entanto, os seus discursos evidenciam que a sua atuação foi fortemente influenciada pela visão da família como potencial disseminador de infeção, demonstrando uma avaliação mais negativa do que positiva desta presença para a manutenção dessa segurança, nem sempre suportada pela evidencia disponível.

Conclusão: As medidas restritivas poderão estar a contribuir para um retrocesso na prestação de cuidados centrados na família do doente hospitalizado. Contudo, há falta de dados a nível nacional que permitam perceber se as famílias em Portugal representam um risco para a segurança nos hospitalizados.

DESCRITORES: Segurança do Paciente, Hospitalização, COVID-19, Gestão da Segurança, Enfermagem de Reabilitação, Enfermagem Famíliar

\section{ABSTRACT}

Introduction: Due to the pandemic by COVID-19, to ensure the safety of health care and to contain its transmission, restrictive measures were taken to keep families away from hospitalized patients. Such measures have implications.

Objective: to analyze, from the speeches of nurses specialized in rehabilitation nursing (EEER), the advantages, disadvantages and contexts of the family's presence in hospitals, in the light of patient safety, in a pandemic context.

Methodology: Qualitative interpretative study based on James Reason's risk model, conducted through semistructured interviews with 6 EEERs chosen for convenience. Content analysis was performed using the Atlas.ti software. and Bardin's methodology.

Results: 17 categories were identified, grouped according to the representation of the family in patient safety: 10 categories in the view of the family that potentiates security failures and 7 within the family as a security barrier.

Discussion: The EEERs consider the presence of the family as very important for hospitalized patients and for safety care, however, their speeches show that their performance was strongly influenced by the view of the family as a potential disseminator of infection, demonstrating a more negative assessment than positive of this presence for the maintenance of this security, not always supported by the available evidence. 
Conclusion: Restrictive measures may be contributing to a setback in the provision of care centered on the hospitalized patient's family. However, there is a lack of data at the national level that allows us to understand whether families in Portugal represent a safety risk in hospitalized patients.

DESCRIPTORS: Patient Safety, Hospitalization, COVID-19, Safety Management, Rehabilitation Nursing, Family Nursing

\section{RESUMEN}

Introducción: Debido a la pandemia por COVID-19, para garantizar la seguridad de la atención de salud y contener su transmisión, se tomaron medidas restrictivas para mantener a las familias alejadas de los pacientes hospitalizados. Tales medidas tienen implicaciones.

Objetivo: analizar, a partir de los discursos de enfermeras especializadas en enfermería rehabilitadora (EEER), las ventajas, desventajas y contextos de la presencia de la familia en los hospitales, a la luz de la seguridad del paciente, en un contexto pandémico.

Metodología: Estudio interpretativo cualitativo basado en el modelo de riesgo de James Reason, realizado a través de entrevistas semiestructuradas con 6 EEER elegidos por conveniencia. El análisis de contenido se realizó utilizando el software Atlas.ti. y la metodología de Bardin.

Resultados: se identificaron 17 categorías, agrupadas según la representación de la familia en la seguridad del paciente: 10 categorías en la visión de la familia que potencializa brechas de seguridad y 7 dentro de la familia como barrera de seguridad.

Discusión: Los EEER consideran muy importante la presencia de la familia para los pacientes hospitalizados y para la seguridad de los cuidados, sin embargo, sus discursos muestran que su desempeño estuvo fuertemente influenciado por la visión de la familia como un potencial diseminador de la infección, demostrando una valoración más negativa. que positivo de esta presencia para el mantenimiento de esta seguridad, no siempre respaldado por la evidencia disponible.

Conclusión: Las medidas restrictivas pueden estar contribuyendo a un retroceso en la prestación de cuidados centrados en la familia del paciente hospitalizado. Sin embargo, faltan datos a nivel nacional que permitan comprender si las familias en Portugal representan un riesgo para la seguridad de los pacientes hospitalizados.

DESCRIPTORES: Seguridad del Paciente, Hospitalización, COVID-19, Administración de la Seguridad, Enfermería en Rehabilitación, Enfermería de la Familia

\section{INTRODUÇÃO}

0 primeiro caso diagnosticado com Corona Virus Desease (COVID-19) aconteceu em Wuhan, China, em dezembro de 2019. Desde então, segundo a Organização Mundial da Saúde (1), ocorreram mais de 237 milhões de casos confirmados de COVID-19 e mais de 4 milhões de mortes resultantes desta doença (2). A pandemia causada pelo coronavírus foi reconhecida como tal pela OMS a 11 de Março de 2020 (3) e é atualmente considerada pelo mesmo organismo como a maior emergência global de saúde pública e responsável por uma extrema pressão nos sistemas de saúde (4).

Desta forma, houve um maior reconhecimento dos riscos para os doentes e a segurança dos cuidados reafirmou-se como um grande foco de preocupação e atuação (5).

A segurança na prestação de cuidados é considerada um fenómeno complexo devido à envolvência de inúmeros fatores, pelo que é essencial a análise dos mecanismos que concorrem para a causalidade do erro. James Reason (6) desenvolveu o modelo do queijo suíço (figura 1) para explicar a dinâmica das falhas de um sistema. Considera que os sistemas são dotados de barreiras, como equipamentos, pessoas ou tecnologias, que estão posicionadas intencionalmente para evitar o erro. Idealmente nas barreiras não haveria falhas, contudo, o autor reconhece que as falhas existem e estas estão representadas pelos buracos nas fatias de queijo suíço. Caracteriza-as como dinâmicas pelo que abrem e fecham em diferentes localizações. A existência de falhas isoladas não implica necessariamente a ocorrência de erro. Este ocorre quando se alinham as falhas de todas as barreiras numa trajetória com risco para a segurança do cliente. Ao considerar este modelo explicativo do mecanismo do erro, a gestão do risco tende a tornarse mais proactiva $(6,7)$. 


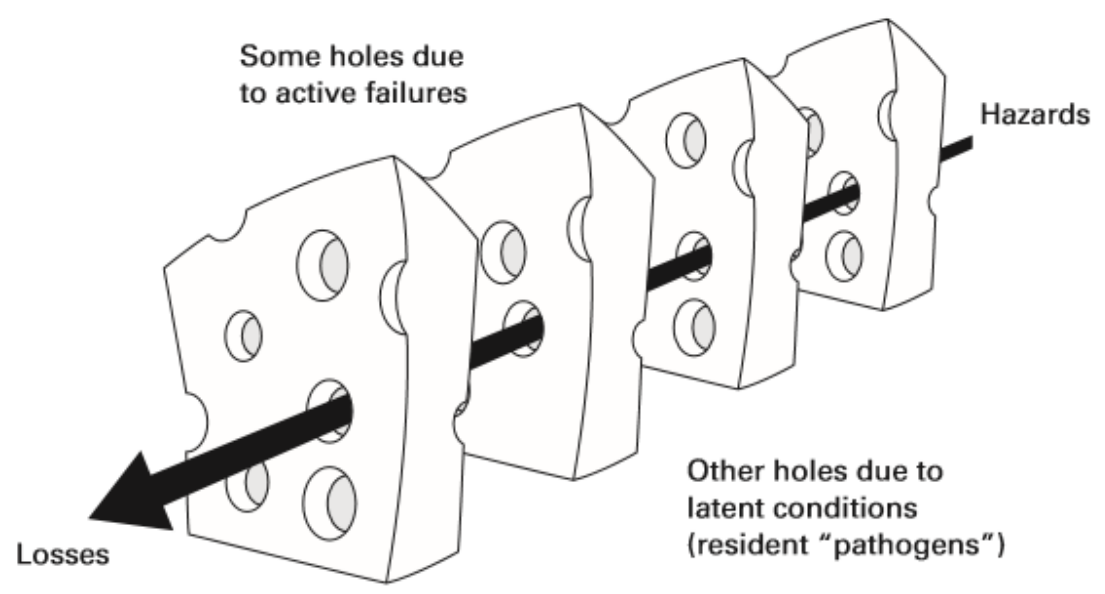

Successive layers of defences, barriers and safeguards

Figura 1 - Modelo de risco do "Queijo Suíço" de James Reason / Fonte: Reason, Carthey (7)

Neste contexto pandémico, no sentido de garantir a segurança dos cuidados de saúde e conter a transmissão de COVID-19 em muitos sistemas de saúde, nomeadamente no Serviço Nacional de Saúde (SNS) Português, entre as medidas adotadas surgiu a restrição a acompanhantes e visitas aos doentes internados em unidades hospitalares, veiculada na Orientação $n^{\circ}$ 038/2020 de 17/12/2020 (8).

Tais medidas trazem implicações para os doentes, famílias, enfermeiros, restantes profissionais de saúde e aos próprios serviços de saúde (9).

Em contraponto, o Cuidado Centrado no Doente e na Família (Patient and Family-Centred Care - PFCC) é uma abordagem que se baseia em parcerias mutuamente benéficas entre prestador de cuidados de saúde, doentes e famílias nos vários contextos de cuidados de saúde (10). Nesta filosofia de cuidados, os doentes e seus familiares definem a sua família e estabelecem como pretendem participar no processo de prestação de cuidados, nomeadamente na tomada de decisão (10). Esta perspetiva baseia-se no reconhecimento dos doentes e familiares como aliados fundamentais para a qualidade e segurança dos cuidados de saúde na medida em que o PFCC leva a melhores resultados em saúde, nomeadamente reduzindo hospitalizações e erros em saúde, melhorando a experiência de cuidados e satisfação dos doente e família e tornando mais eficaz a gestão dos recursos $(10,11)$.

Para tal importa ter presente o conceito de família sob várias perspetivas: legal, biológica, sociológica ou psicológica. Do ponto de vista da prestação de cuidados de enfermagem, uma das definições adotadas é a que considera a família como "dois ou mais indivíduos, que dependem um do outro para efeitos de apoio emocional, físico e económico. Os membros da família são auto-definidos." (12).

Eventos de saúde e a família afetam-se mutuamente sendo que, quando um elemento da família adoece, todos os outros elementos da mesma são afetados (12). Constata-se assim que eventos de saúde têm um grande potencial de risco para a família, particularmente no seu funcionamento, o que leva a considerá-la população em risco. População em que os enfermeiros têm a responsabilidade de intervir, nesses períodos críticos, no sentido de apoiar no desenvolvimento da capacidade de adaptação, reorganização e manutenção da saúde no seu global (12).

Em consonância com o exposto, na definição das competências do Enfermeiro de cuidados gerais, a Ordem dos Enfermeiros define que o processo de tomada de decisão deve fundamentar-se numa abordagem sistémica e sistemática da pessoa, família e comunidade, e estabelece critérios de competência, nomeadamente para apoiar pessoas, famílias e comunidades nos seus desafios de saúde numa ótica holística (13) . A relação terapêutica estabelecida pelo enfermeiro não se esgota na parceria com o cliente, mas expande-se à família e comunidade.

Por sua vez, e de acordo com o Regulamento das Competências Específicas do Enfermeiro Especialista em Enfermagem de Reabilitação (2010), o enfermeiro especialista em enfermagem de reabilitação “... utiliza técnicas específicas de reabilitação e intervém na educação dos clientes e pessoas significativas, no planeamento da alta, na continuidade dos cuidados e na reintegração das pessoas na família e na comunidade, proporcionando-lhes assim, o direito à dignidade e à qualidade de vida." (14).

O objetivo deste estudo é analisar, a partir dos discursos dos enfermeiros especialistas em enfermagem de reabilitação (EEER), as vantagens, desvantagens e contextos da presença da família durante o internamento hospitalar, à luz da segurança do doente, durante a pandemia de COVID-19.

\section{METODOLOGIA}

Considerando que o objeto em análise se apresenta com uma natureza complexa e dinâmica e perante a escassez de estudos nesta área, desenvolveu-se um estudo qualitativo, interpretativo, baseado no Modelo do risco de James Reason (7). 
O método de amostragem é não probabilística intencional, os sujeitos de investigação deste estudo são enfermeiros em funções durante o período de pandemia COVID-19 em 3 hospitais nacionais do norte, selecionados por conveniência por serem os mais acessíveis e simultaneamente cumprirem os critérios de inclusão pré-determinados (15). Estes critérios são: ter especialidade em Enfermagem de Reabilitação, ter experiência em serviços de internamento hospitalar de adultos, estar a exercer funções no momento da entrevista. Do universo de 11 enfermeiros entrevistados, com diferentes especialidades, constituímos a população deste estudo apenas com 6 enfermeiros, os que preenchiam o critério da especialidade em Enfermagem de Reabilitação: 5 enfermeiras e um enfermeiro, com idades entre os 34 e 62 anos (média de 43 anos), com tempo de experiência entre 8 e 39 anos (média de 20 anos) sendo que 4 dos entrevistados são mestres, dos quais, dois mestres em reabilitação, 2 em Ciências de Enfermagem e 1 em cuidados paliativos.

A colheita de dados realizou-se com entrevistas semiestruturadas individuais com questões abertas que "permitem fazer ressaltar os pontos de vista dos participantes, e ter uma ideia mais precisa do que constitui a sua experiência" (15). As entrevistas decorreram entre junho e setembro de 2020 via zoom. As questões cujos dados são analisados nesta fase foram:

- Que avaliação faz da presença da família junto dos doentes internados?

- O que vê de positivo e de negativo na restrição de visitas?

- Como considera a família para a segurança dos cuidados?

Após transcritas, as entrevistas foram enviadas ao respetivo entrevistado para que fossem validadas.

A análise de conteúdo fez-se com recurso à metodologia de Bardin (16) que compreende três fases: a pré-análise, a exploração do material e por último o tratamento dos resultados obtidos e interpretação (16).

$\mathrm{Na}$ leitura exploratória procuraram-se conteúdos comuns, o que originou a identificação de áreas temáticas denominadas categorias, sempre com o Modelo de James Reason $(6,7)$ como referencial teórico orientador deste processo. Como instrumento de auxílio na codificação e categorização das entrevistas recorreu-se ao software Atlas.ti ${ }^{\circledast}$. As unidades de análise foram identificadas a partir dos fragmentos das entrevistas que, posteriormente, foram reunidas em grandes áreas temáticas as quais possibilitaram o desenvolvimento dos resultados deste estudo.

Foram respeitados os princípios éticos e legais. 0 estudo foi autorizado pela Comissão de Ética conjunta do Centro Hospitalar e Universitário do Porto e Instituto Ciências Biomédicas Abel Salazar (ICBAS) da Universidade do Porto (UP). Todos os procedimentos realizados com os participantes respeitaram o anonimato, a confidencialidade e o consentimento informado.

\section{RESULTADOS}

Da análise de conteúdo das entrevistas aos EEER, foram identificadas dezassete categorias que foram agrupadas em duas grandes áreas temáticas de acordo com o posicionamento dessas categorias no modelo de risco de James Reason (7) (figura 1): Família como potencializadora de falhas de segurança e Família como barreira de segurança (figura 2 e 3).

\section{Família como potencializadora de falhas de segurança}

Dentro da visão da presença da família no hospital enquanto potencializadora de falhas de segurança, foram identificadas 10 categorias: Falta de capacidade das famílias, Riscos na alimentação, Risco de queda, Risco de infeção COVID, Risco de infeção, Falta de informação das famílias, Família não colaborante, Família desrespeitadora de indicações de enfermagem, Família desrespeitadora das normas, Presença das famílias dificultadora de cuidados (figura 2).

A falta de informação das famílias foi muito referida pelos participantes como algo frequente nas suas realidades de cuidados: "Nós temos famílias que atrapalham muito, infelizmente, mas acho que é porque elas não sabem, é mesmo porque não estão informadas." (E3). Neste contexto pandémico, foi referido que sobre medidas especificas de contenção da transmissão de COVID-19, há muita falta de informação: “...a população em geral ainda tem bastante desconhecimento sobre algumas medidas restritivas ou de controlo de infeção daquilo que penso que pode passar ainda pela necessidade de educação dos familiares." (E5). Houve ainda quem defendesse que "....as pessoas na escola deviam ter um curso sobre como estar num hospital, porque toda a gente um dia vai ter de vir para o hospital e se na escola falassem sobre isso já era a formação cívica no fundo..." (E1).

Um dos participantes referiu ainda que há famílias que não têm capacidade para gerir e cumprir as informações dadas pela equipa de enfermagem: “...muitas vezes as informações que nós damos são demasiado para a capacidade deles reproduzirem as nossas indicações de segurança...” (E1) e desta forma podem representar um risco para segurança do seu familiar internado. 


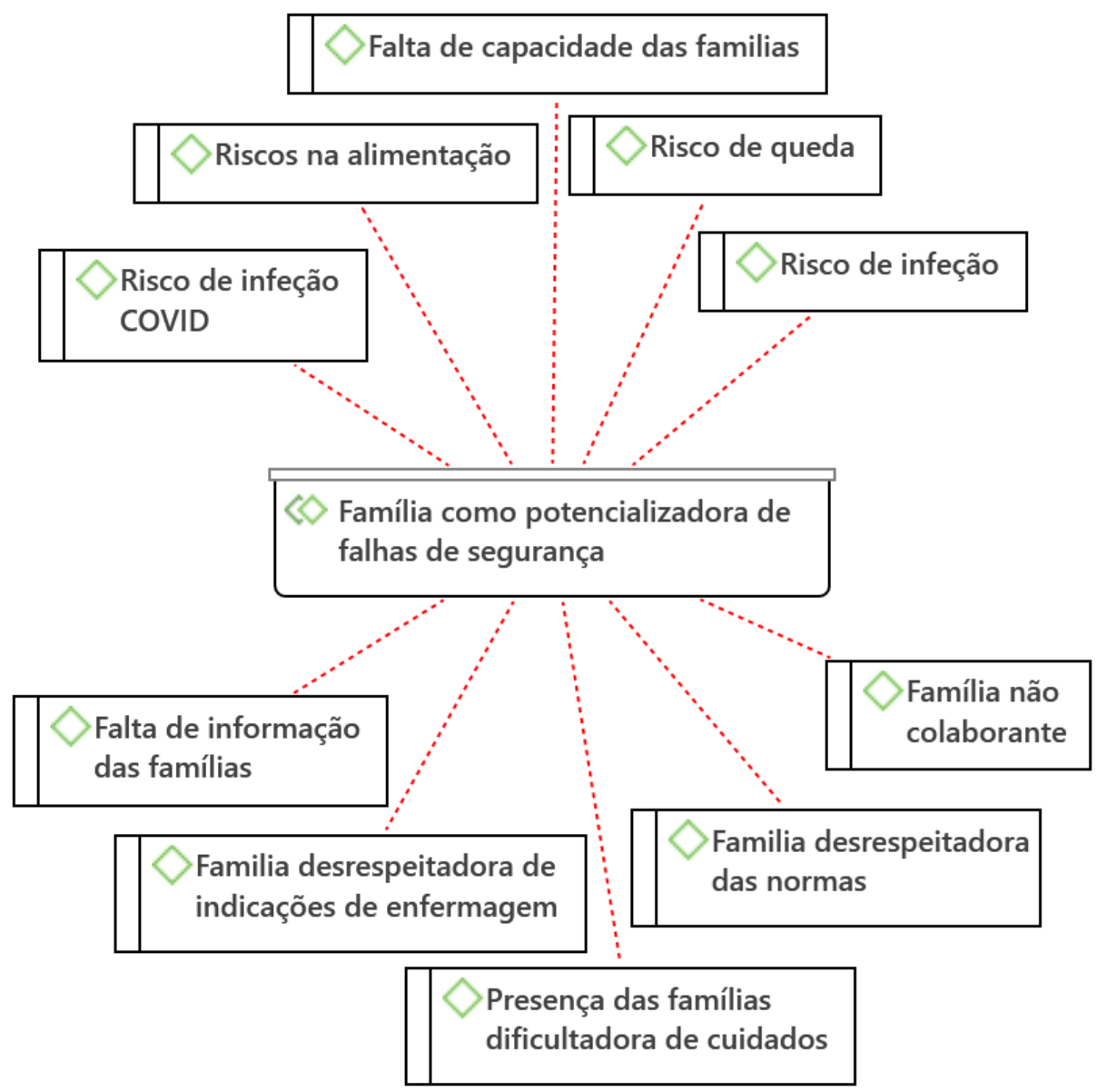

Figura 2 - Categorias identificadas no âmbito da Família como potencializadora de falhas de segurança

A Família desrespeitadora das normas foi também referida nas entrevistas como uma preocupação para os enfermeiros no que diz respeito à segurança da pessoa internada: "É lógico que se a família não tiver uma conduta adequada, se não respeitar as normas de controlo de infeção, a família torna-se insegura para a prestação se cuidados." (E2)

Outra fonte de preocupação apontada pelos EEER foi o desrespeito que as famílias apresentam das indicações dadas pela equipa de enfermagem: "Eu acho que as famílias só põem em causa a segurança dos utentes se não estiverem de acordo com a nossa prestação de cuidados. Por exemplo, eu tenho o doente imobilizado, até explico porque é que ele está imobilizado, e o familiar mesmo assim retira a imobilização e o doente arranca a sonda nasogástrica. Isto acontece com muita frequência e nestas situações as famílias poem em causa a prestação de cuidados e em risco a segurança do doente."(E3).

Também identificaram que há famílias não colaborantes: “... quando adquirem uma postura dificultadora na prestação de cuidados, de criar obstáculos, de criar stress no próprio doente, acho que aí faço uma avaliação negativa na presença da família... É que por vezes há famílias tão difíceis para nós que nos levam a pensar que o COVID veio ajudar-nos a evitar essas situações difíceis." (E3).

Houve também quem identificasse a presença das famílias como dificultadora da prestação de cuidados: "Eu sei que isto pode ser considerado injusto com as famílias, mas esta restrição foi positiva na medida em que veio simplificar os nossos cuidados... Não somos tantas vezes interrompidos com questões por parte da família, o que é normal e eu no lugar deles faria o mesmo... não temos de fazer esta gestão em função da família."(E3).

Houve unanimidade nos discursos sobre a avaliação da presença da família como risco para o controlo de infeção: “...o nível do controle de infeção, eles não têm noção das coisas e por isso não têm cuidado, não lavam as mãos, pousam os pertences em qualquer lado." (E4); "Nós também temos problemas com aqueles familiares que, em vez de virem visitar o seu doente, estão a conversar por dentro da cama ao lado e interferem com o controlo de infeção e esses problemas efetivamente também vão surgindo..." (E1).

Especificamente sobre o risco de transmissão de COVID, mais uma vez, a presença da família no meio hospitalar, foi referida como potencializadora de falhas na segurança do doente: "Os nossos doentes são testados efetivamente e os familiares não são testados e é um risco."(E1), pelo que a restrição de visitas foi avaliada positivamente: "Positivo, a não contaminação dos profissionais e dos doentes." (E6).

A presença da família também foi referida como potencializadora do risco de queda: "Nós temos muitas quedas no serviço, temos receio que os familiares levantem os doentes, que os deixem cair ou até caiam os dois...Quando vinha 
a família, nós explicávamos à família e tirávamos o cinto porque achávamos desnecessário estar imobilizado estando acompanhado, mas esse doente caiu mais vezes com a família do que connosco." (E4).

Riscos na alimentação foram também identificados pelos participantes como incrementados pela presença das famílias: "Muitas vezes a família está associada a eventos relacionados com a alimentação.”(E4).

\section{Família como barreira de segurança}

No âmbito do reconhecimento da presença da família no meio hospitalar como barreira de segurança, foram identificadas sete categorias: Comunicação mais efetiva com a família, Promotora da adesão ao regime terapêutico, Promotora de segurança hospitalar, Parceira na segurança hospitalar, Família colaborante, Família informada, Preparação de alta segura (figura 3).

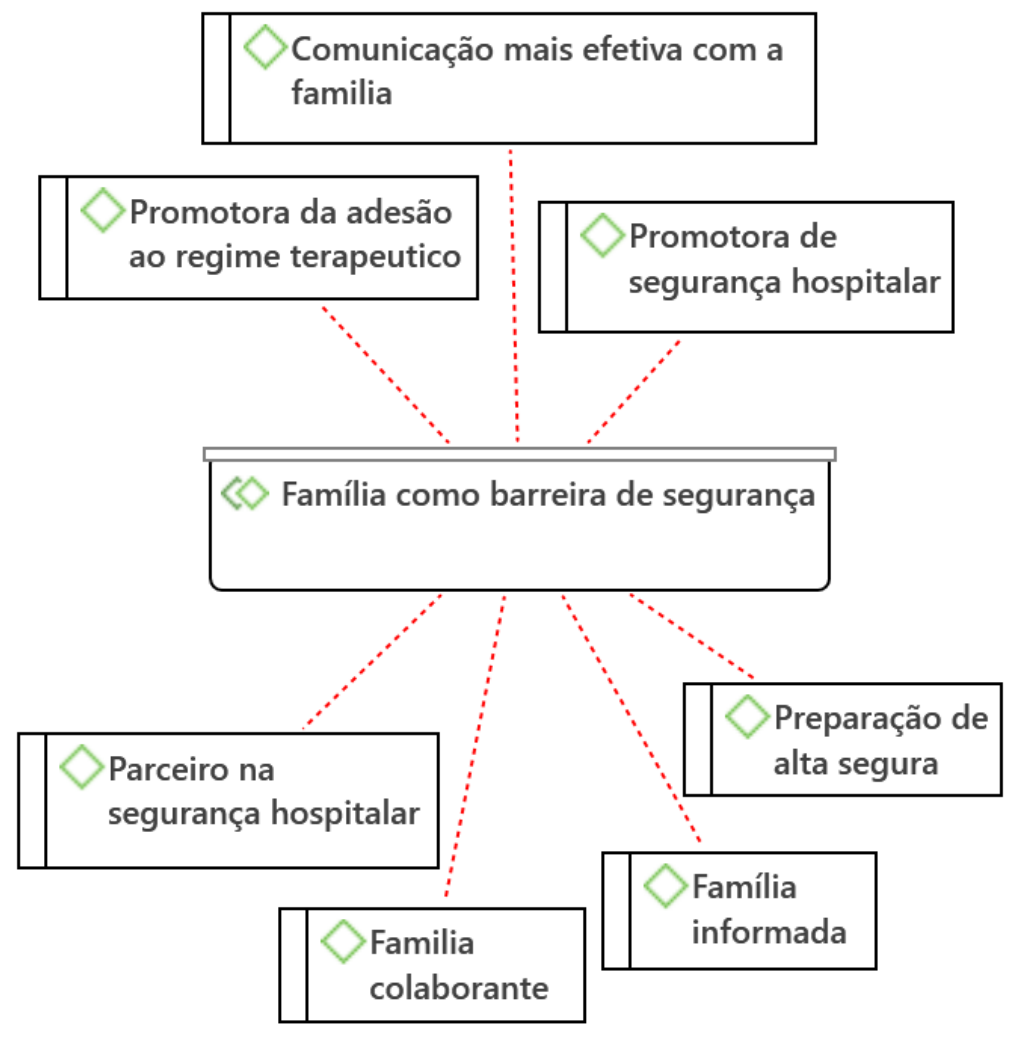

Figura 3 - Categorias identificadas no âmbito da Família como barreira de segurança

A presença da família foi avaliada como fator de segurança na medida em que a comunicação com a família se torna mais efetiva: "É vantajoso em termos emocionais e em termos de passagem de informação mais efetiva com a família, o regresso a casa mais preparado..." (E1).

E que uma família colaborante é uma mais-valia para a segurança dos cuidados: "Se a família quiser ter uma postura de colaboração connosco, de aprendizagem, de enriquecimento de estratégias, para depois mais tarde usarem no cuidado no domicílio, acho que é extremamente positiva a presença das famílias.” (E3).

Da mesma forma uma família informada é considerada barreira protetora no que se refere à segurança do doente internado: "Se todas as normas de conduta forem respeitadas, se for explicado, ensinado à família como devem se comportar no hospital, eu acho que a presença da família tem mais vantagens do que desvantagens." (E2).

Com a restrição da presença de famílias no hospital, verificaram a sua importância na adesão ao regime terapêutico: “...tanto para o utente como para profissionais, foi muito negativo, levou ao isolamento à solidão, à falta de relação de afeto e até resistência na adesão aos tratamentos." (E2)

Foi também considerado pelos participantes que a presença da família em ambiente hospitalar é fundamental para a preparação de uma alta segura: "Penso que a presença da família também é importante no sentido de consciencializar as reais necessidades de cuidado do doente, o que faz com que se consiga prever dificuldades e necessidades de ajustar a prestação de cuidados, por exemplo, se for o caso, aquando do regresso a casa. Portanto, em termos de planeamento de alta e continuidade de cuidados, na minha opinião a presença da família é muito importante." (E5).

A família foi referida por alguns participantes como promotora de segurança do doente: “É muito benéfica, quer para o doente quer para o profissional, uma segurança na prestação de cuidados.”(E6), “...acho que a família apresenta sempre vantagens em poder estar junto dos utentes/doentes. Vantagem não só pela promoção da Segurança junto 
do utente, a família também é um incentivo à adesão ao tratamento, é um apoio em fim de vida, um apoio em situações de dor e angústia extrema." (E2).

A família chegou mesmo a ser considerada parceira na segurança hospitalar: "É um elemento importante, essencial para que haja mais segurança e mais qualidade." (E1).

\section{DISCUSSÃO}

$\mathrm{Na}$ análise das entrevistas foi possível verificar que todos os participantes consideram de forma genérica que é importante a presença da família junto dos seus familiares internados, como se pode verificar nas palavras mais frequentes dos entrevistados: família, ser, doente, cuidado, entre outras (figura 4). Contudo, no que se refere a essa presença em confronto com a segurança do doente, apresentam posicionamentos díspares. Se para alguns EEER a família é identificada como barreira de segurança e, por conseguinte, o envolvimento da família na segurança do doente hospitalizado é vantajoso, para outros, ou até para os mesmos, alega-se que a família é potencializadora de falhas de segurança. Os seus discursos apontaram mais preocupações do que vantagens nesta presença, o que se demonstra na distribuição das categorias e volumes de discurso: dez categorias identificadas no âmbito da família como potencializadora de falhas de segurança e sete categorias no âmbito da família como barreira de segurança e também na maioria das palavras identificadas como mais frequentes: infeção, problema, controlar, positivar, causar (figura 4).

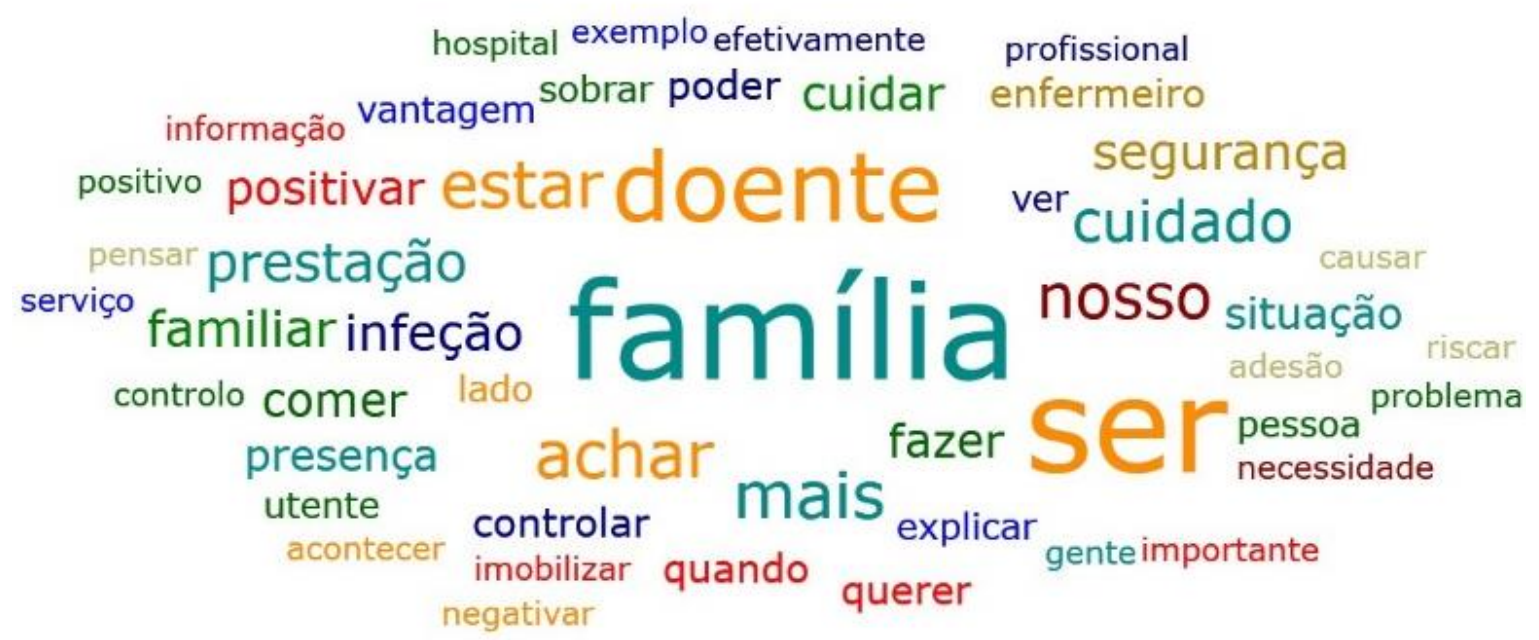

Figura 4 - Nuvem de palavras identificadas de acordo com a sua frequência nos discursos dos entrevistados

A evidência tem registado achados semelhantes: por um lado a presença da família é vista pelos enfermeiros como uma mais valia para a segurança do doente, por outro, perante medidas mais concretas como o alargamento do horário de visitas, alegam que a segurança dos doentes fica em risco, que esta medida os desprotege, veem a família como um elemento perturbador e temem um aumento da carga de trabalho (17).

Quanto à colaboração da família na segurança dos doentes, estudos têm demonstrado que as famílias se esforçam para salvaguardar a segurança dos seus familiares hospitalizados o que fundamenta as categorias identificadas no âmbito da família como barreira de segurança, particularmente: Promotora de segurança hospitalar, Parceira na segurança hospitalar, Família colaborante (17) contrapondo-se às categorias identificadas em sentido contrário: Família não colaborante, Família desrespeitadora de indicações de enfermagem, Família desrespeitadora das normas.

Sobre o risco de infeção, principal fundamento para a restrição de visitas hospitalares e principal risco apontado nos discursos dos EEER à presença das famílias em ambiente hospitalar, patente nas categorias Risco de infeção COVID, Risco de infeção, a evidência demonstra que as famílias procuram atuar de acordo com as recomendações para a prevenção do risco de infeção mas que se sentem pouco preparadas e acompanhadas pelos profissionais de saúde para colaborar a este nível (17). Estudos anteriores indicaram que a presença da família em cuidados intensivos de adultos não está associada a aumento das taxas de infeção (18). Já durante a atual crise pandémica, a Holanda abriu 26 lares de idosos aos familiares e não houve registo de novas infeções (19). Na verdade, a literatura disponível não demonstra um papel substancial das visitas na transmissão da COVID-19 nos hospitais (20).

Sobre esse e restantes riscos apontados pelos enfermeiros, os dados apontam para que possa haver uma relação positiva entre a família e a segurança do doente. No entanto, a relação entre o envolvimento do doente e da família em questões de segurança dos cuidados de saúde e a redução de danos não é bem compreendida, sendo que os achados disponíveis na literatura atual são limitados (17). 
A categoria Comunicação mais efetiva com a família no âmbito da família como barreira de segurança ganha particular relevo quando falamos em doentes idosos, muitas vezes com alterações cognitivas associadas, pelo que os familiares presentes podem estar mais atentos a mudanças a este nível e fornecer informações muito importantes à prestação de cuidados (21).

Diversos estudos realizados sobre questões de segurança dos cuidados de saúde, apontam para a necessidade de melhorar a comunicação entre os profissionais de saúde e a família (17). Sobre esta dinâmica família-enfermeiro, foram descritos por Doherty (22) cinco níveis de envolvimento de prestação de cuidados à família. Nos discursos dos participantes foi possível verificar que, com a restrição de visitas, o nível de envolvimento de prestação de cuidados à família é o nível 1 , em que os cuidados de enfermagem dão ênfase mínima à família estabelecendo contactos apenas por razões práticas ou legais como a assinatura de um consentimento para realização de exame (22). Tal é possível verificar, nomeadamente na categoria "Presença da família dificultadora de cuidados", quando foi referido que a restrição de visitas foi positiva porque "Quando temos as famílias, temos que adaptar às condições das famílias. Por isso é lado positivo porque simplifica a nossa prestação de cuidados."(E3). Contudo, quando as famílias estão presentes no ambiente hospitalar o nível de envolvimento nos cuidados prestados à família evidenciado nos discursos é maioritariamente de nível 2 em que os cuidados de enfermagem à família são generalistas e dizem respeito a aconselhamentos ou ensinos sobre determinada temática de saúde: "se for explicado, ensinado a família como devem se comportar no hospital, eu acho que a presença da família tem mais vantagens do que desvantagens." (E2). O nível 3 , em que a intervenção de enfermagem implica o apoio à família permitindo que esta expresse emoções e conhecimentos acerca da dinâmica e stress familiares reativos a situação de saúde/doença encorajando as estratégias de coping foi referido no âmbito avaliação emocional da família, sem que fossem referidas intervenções especificas: “É vantajoso em termos emocionais e em termos de passagem de informação mais efetiva com a família,..." (E1).

Importa mencionar que existem mais dois níveis de intervenção de enfermagem à família. No nível 4 , o enfermeiro atua de modo sistemático na avaliação e intervenção na família o que implica um conhecimento específico dos sistemas de família e dos sistemas que interagem com a mesma. No nível 5 , requer-se que o enfermeiro detenha um elevado grau de conhecimentos e competências para intervir na família como é o caso do enfermeiro especialista em saúde mental e psiquiatria ou com formação em terapia familiar $(12,22)$.

Assim, verifica-se que esta restrição das visitas ao doente hospitalizado limita os cuidados de enfermagem à família e promove um retrocesso no nível de envolvimentos em que os enfermeiros prestam cuidados à família. Ainda sobre o desempenho dos EEER durante esta pandemia, importa referir que um estudo realizado também a nível nacional verificou que um número significativo de EEER prestou apenas cuidados gerais durante o primeiro pico pandémico de COVID-19 devido a necessidades emergentes (23). Também se constatou uma diminuição da satisfação com os cuidados prestados a par com uma elevada percentagem de vivencias de desafios éticos e deontológicos (23). Estes achados podem, também eles, contribuir para um retrocesso na qualidade dos cuidados e na prestação de cuidados à família do doente hospitalizado, como referido anteriormente.

Além disso, os discursos dos EEER evidenciam que a sua atuação foi fortemente influenciada pelo entendimento da família enquanto potencial disseminador de infeção. Apesar disso, a evidencia demonstra que iniciativas mais estruturadas de envolvimento da família parecem ter resultados positivos na segurança dos cuidados (17). A segurança dos cuidados e a presença da família não são incompatíveis desde que sejam cumpridas as recomendações nesse sentido (24). Recentemente, a OMS publicou Final draft Global Patient Safety Action Plan 2021-2030 onde, entre os princípios orientadores e como um dos objetivos estratégicos, surge o envolvimento dos doentes e familiares como parceiros em cuidados seguros (5). Refere ainda que a segurança dos cuidados de saúde depende do envolvimento do doente, familiares, cuidadores e comunidades como parceiros nos diversos níveis de cuidados, desde a formulação de políticas e planeamento até a supervisão do desempenho, consentimento totalmente informado e tomada de decisão compartilhada (5). A família é considerada insubstituível no contexto dos cuidados, é quem melhor conhece a história de saúde do doente, pode contribuir na observação e vigilância do doente, alertar para necessidades que surjam de novo e podem ser os olhos e os ouvidos do sistema (5). Para tal é necessário envolver e capacitar as famílias nesse sentido (5) devendo a mesma ser vista como uma parceira na segurança dos cuidados como referido por alguns participantes.

\section{CONCLUSÃO}

A conceptualização do Cuidado Centrado no Doente e na Família tem vindo a desenvolver-se e a ganhar espaço ao demonstrar valor na prática de cuidados. Contudo, devido às medidas restritivas de segurança aplicadas, nomeadamente ao nível hospitalar durante e devido á pandemia por COVID-19, surge a ameaça de uma estagnação ou até mesmo involução nesta área.

É de extrema importância refletir sobre este afastamento das famílias do meio hospitalar.

Foi importante perceber, aqui e a partir dos discursos dos EEER, as vantagens, desvantagens e contextos da presença da família durante o internamento hospitalar, à luz da segurança do doente, durante a pandemia de COVID-19.

Perante a análise dos dados, podemos verificar que os EEER consideram a presença da família como muito importante para doentes internados num hospital e até para a segurança dos cuidados. Todavia, os seus discursos evidenciam que a sua atuação foi fortemente influenciada pela visão da família como potencial disseminador de infeção, 
demonstrando uma avaliação mais negativa do que positiva desta presença para a manutenção desta segurança, entendimento nem sempre suportados pela evidência disponível.

Ainda assim, a inexistência de estudos sobre este tema a nível nacional, não permite perceber se as famílias em Portugal representam um risco para a segurança dos seus familiares hospitalizados pelo que são necessários mais estudos sobre este tema. Na mesma linha de investigação, será importante desenvolver investigação no sentido de perceber que estratégias os enfermeiros e as instituições devem adotar para potenciar o papel das famílias na segurança do doente internado.

\section{REFERÊNCIAS BIBLIOGRÁFICAS}

1. World Health Organization. Corona vírus disease (COVID-19) Situation Dashbord: WHO; 2020 [Available from: https: //covid19.who.int.

2. Chen NS, Zhou M, Dong X, Qu J, Gong F, Hang Y. Epidemiological and clinical characteristics of 99 cases of 2019 novel coronavírus pneumonia in Wuhan, China: adescriptive study. The Lancet 2020;395(10223):507-13.

3. World Health Organization. WHO Director-General's opening remarques at themedia briefing on COVID-19 - 11 March 2020: WHO; 2020 [Available from: https://www.who.int/director-general/speeches/detail/who-director-general-s-opening-remarks-at-the-media-briefing-oncovid-19---11-march-2020.

4. Lai J, Ma S, Wang Y, et al. Factors associated with mental health outcomes among health care workers exposed to coronavirus disease 2019. JAMA network open. 2020;3(3):e203976-e.

5. World Health Organization. Final draft Global Patient Safety Action Plan 2021-2030: towards eliminating avoidable harm in health care. Geneva, Switzerland: WHO; 2021.

6. Reason J. Human error: models and management. British Medical Journal 2000;320(7237):768-70.

7. Reason JT, Carthey J, Leval MR. iagnosing "vulnerable system syndrome": an essential prerequisite to eVective risk management. Quality in Health Care. 2001;10:ii21-ii5.

8. DIreçao Geral da Saúde. Orientação n 038/2020 de 17/12/2020 - COVID-19: Acompanhantes e visitas nas Unidades Hospitalares. Lisboa2020. p. $1-5$.

9. Meiers SJ, Swallow V. COVID-19 Pandemic and Family Nursing: IFNA President and President-Elect Offer a Message to Members USA: International Family Nursing Association; 2021 [Available from: https://internationalfamilynursing.org/2020/03/27/covid-19-pandemic-ifnapresident-and-ifna-president-elect-offer-a-message-to-members/.

10. Johnson BH, Abraham MR. Partnering with Patients, Residents, and Families: A Resource for Leaders of Hospitals, Ambulatory Care Settings, and Long-Term Care Communities. Institute for Patient- and Family-Centered Care; 2012.

11. Clay AM, Parsh B. Patient- and family-centered care: It's not just for pediatrics anymore. AMA J Ethics. 2016;18(1):40-4.

12. Hanson SMH. Enfermagem de cuidados de saúde à familia : teoria, prática e investigação. $2^{\mathrm{a}}$ ed. Loures Lusociência; $2005.497 \mathrm{p}$.

13. Diário da República n. ${ }^{\circ}$ 79/2015, Série II de 2015-04-23: Regulamento do Perfil de Competências do Enfermeiro de Cuidados Gerais, (2015).

14. Ordem dos Enfermeiros. Regulamento das Competências Especificas do Enfermeiro Especialista em Enfermagem de Reabilitação. Lisboa: Ordem dos Enfermeiros; 2010.

15. Fortin M-F. Fundamentos e Etapas no Processo de Investigação. Loures2009. 618 p.

16. Bardin L. Análise de conteúdo. Lisboa: Edições 70; 2000.

17. Correia TSP, Martins MM, Barroso FF. The Family and Safety of the Hospitalized Patient: An Integrative Literature Review. Portuguese Journal of Public Health. 2020;38:1-12.

18. Bishop SM, Walker MD, Spivak IM. Family presence in the adult burn intensive care unit during dressing changes. Crit Care Nurs. 2013;33(1):1424.

19. Verbeek H, Gerritsen DL, Backhaus R, Boer BS, Koopmans R, Hamers J. Allowing visitors back in the nursing home during the COVID-19 crisis: A Dutch national study into first experiences and impact on well-being. Journal of the American Medical Directors Association. 2020;21(7):9004.

20. Munshi L, Evans G, Razak F. The case for relaxing no-visitor policies in hospitals during the ongoing COVID-19 pandemic. CMAJ. 2020.

21. Ehlenbach WJ, Hough CL, Crane PK, Haneuse SJ, Carson SS, Curtis JR, et al. Association between acute care and critical illness hospitalization and cognitive function in older adults JAMA. 2010;303(8):763-70.

22. Doherty WJ. Family Interventions in Health Care. Family Relations. 1985;34:129-37.

23. Padilha JM, Silva RP. Impacte da pandemia por COVID-19 nos Enfermeiros de Reabilitação portugueses. Revista Portuguesa de Enfermagem de Reabilitação. 2020;3(2):102-7.

24. Institute for Patient-And Family-Centered Care. Family presence during a pandemic: Guidance for decision-making. Bethesda, USA: IPAFCC; 2021.

\section{DIVULGAÇÕES ÉTICAS}

Contribuição dos autores:

Conceptualização: TC, MMM, FB;

Metodologia: TC, MMM;

Validação: MMM, FB;

Análise formal: TC, MMM, FB;

Investigação: TC;

Tratamento de dados: TC, MMM, FB;

Preparação do rascunho original: TC;

Redação e edição: TC;

Revisão: MMM, FB;

Todos os autores leram e concordaram com a versão publicada do manuscrito.

Financiamento: 
Este trabalho não recebeu nenhuma contribuição financeira ou bolsa.

Comissão de Ética:

Estudo autorizado pela Comissão de Ética conjunta do Centro Hospitalar e Universitário do Porto e Instituto Ciências Biomédicas Abel Salazar (ICBAS) da Universidade do Porto (UP) de 22/05/2020 (documento 2020/CE/P010).

Declaração de consentimento informado:

0 consentimento informado por escrito para publicar este trabalho foi obtido dos participantes.

Agradecimentos:

Os autores agradecem o tempo, disponibilidade e a colaboração dos enfermeiros que aceitaram participar neste estudo.

Conflitos de interesse:

Os autores não declaram nenhum conflito de interesses.

Proveniência e revisão por pares:

Não comissionado; revisto externamente por pares.

(c) Autor (es) (ou seu (s) empregador (es)) e APER/RPER 2021. Reutilização permitida de

(c) (1) $\Theta \Theta$ acordo com CC BY-NC-ND. Nenhuma reutilização comercial. 